\title{
Phenotypic Characteristics of PD-1 and CTLA-4 Expression in Symptomatic Acute Hepatitis A
}

Hyosun Cho*, Hyojeung Kang ${ }^{\dagger}$, Chang Wook Kim${ }^{\ddagger}$, Hee Yeon $\mathrm{Kim}^{\ddagger}$, Jeong Won Jang ${ }^{\ddagger}$, Seung Kew Yoon ${ }^{\ddagger}$, and Chang Don $\operatorname{Lee}^{\ddagger}$

*Department of Pharmacy, Duksung Women's University College of Pharmacy, Seoul, 'Department of Pharmacy, Kyungpook National University College of Pharmacy, Daegu, and ${ }^{\ddagger}$ Department of Internal Medicine, The Catholic University of Korea College of Medicine, Seoul, Korea

Background/Aims: The immunoregulatory molecules programmed death 1 (PD-1) and cytotoxic T lymphocyte-associated antigen 4 (CTLA-4) are associated with the dysfunction of antiviral effector T-cells, which leads to T-cell exhaustion and persistent viral infection in patients with chronic hepatitis $\mathrm{C}$ and chronic hepatitis $\mathrm{B}$. Little is known about the role of PD-1 and CTLA-4 in patients with symptomatic acute hepatitis A (AHA). Methods: Peripheral blood mononuclear cells were isolated from seven patients with AHA and from six patients with nonviral acute toxic hepatitis (ATH) during the symptomatic and convalescent phases of the respective diseases; five healthy subjects acted as controls. The expression of PD-1 and CTLA-4 on T-cells was measured by flow cytometry. Results: PD-1 and CTLA-4 expression during the symptomatic phase was significantly higher in the Tcells of AHA patients than in those of ATH patients or healthy controls (PD-1: $18.3 \%$ vs $3.7 \%$ vs $1.6 \%$, respectively, $p<0.05$; CTLA-4: $23.5 \%$ vs $6.1 \%$ vs $5.9 \%$, respectively, $p<0.05$ ). The levels of both molecules decreased dramatically during the convalescent phase of AHA, whereas a similar pattern was not seen in ATH. Conclusions: Our findings are consistent with a viral-protective effect of PD- 1 and CTLA- 4 as inhibitory molecules that suppress cytotoxic T-cells and thereby prevent the destruction of virus-infected hepatocytes in AHA. (Gut Liver 2016;10:288-294)

Key Words: Acute hepatitis A; Cytotoxic T lymphocyte-associated antigen 4; Programmed death 1

\section{INTRODUCTION}

Acute hepatitis A (AHA) is a viral infectious disease of the liver caused by hepatitis A virus (HAV). It is usually transmitted through the fecal-oral route and contaminated drinking water. ${ }^{1}$ The incidence of HAV infection is high in regions with poor standards of hygiene. Initial symptoms of AHA are nonspecific and include fever, headache, fatigue, and often jaundice. ${ }^{2}$ Unlike hepatitis B virus (HBV) or hepatitis C virus (HCV), HAV infection produces a self-limited, nonchronic disease. However, the mechanism underlying HAV-mediated disease is poorly understood.

Programmed death 1 (PD-1) and cytotoxic T lymphocyte antigen 4 (CTLA-4) are receptors in the CD28 family of costimulatory molecules but they provide inhibitory signals to T-cells. ${ }^{3}$ PD-1 and CTLA-4 expression on HCV-specific T-cells results in impaired effector T-cell function, ${ }^{4}$ while PD-1 expression was shown to correlate positively with HBV viremia. ${ }^{5}$ Similarly, human immunodeficiency virus (HIV) persists in dysfunctional antiviral effector T-cells with increased PD-1 and/or CTLA-4 expression. ${ }^{6-8}$ By contrast, little is known about the roles of PD-1 and CTLA-4 in patients with symptomatic AHA.

In this study, the expression of PD-1 and CTLA-4 during the symptomatic and convalescent phases of acute hepatitis was examined in T-cells from patients with symptomatic AHA and in those from patients with nonviral acute toxic hepatitis (ATH).

\section{MATERIALS AND METHODS}

\section{Patients}

Patients were recruited from the Department of Internal Med-

\footnotetext{
Correspondence to: Chang Wook Kim

Department of Internal Medicine, Uijeongbu St. Mary's Hospital, The Catholic University of Korea College of Medicine, 271 Cheonbo-ro, Uijeongbu 480-717, Korea

Tel: +82-31-820-3997, Fax: +82-31-820-5291, E-mail: cwkim@catholic.ac.kr

Received on September 22, 2014. Revised on March 20, 2015. Accepted on April 6, 2015. Published online September 9, 2015

pISSN 1976-2283 eISSN 2005-1212 http://dx.doi.org/10.5009/gnl14368

Hyosun Cho and Hyojeung Kang contributed equally to this work as first authors.

@ This is an Open Access article distributed under the terms of the Creative Commons Attribution Non-Commercial License (http://creativecommons.org/licenses/by-nc/4.0) which permits unrestricted non-commercial use, distribution, and reproduction in any medium, provided the original work is properly cited.
} 
icine of Uijeongbu St. Mary's Hospital, Korea. Informed consent was obtained from all study participants and the study was approved by the hospital's Institutional Review Board. Three groups of patients were evaluated: those with symptomatic acute hepatitis due to HAV infection (seven patients), those with nonviral ATH (six patients), and five healthy controls. Exclusion criteria were excessive alcohol consumption, HBV, HCV, or HIV infection, autoimmune liver disease, Wilson's disease, or significant medical comorbidities. AHA was diagnosed based on the presence of serum IgM anti-HAV. Nonviral ATH was diagnosed based on the absence of viral markers (IgM anti-HAV, hepatitis B surface antigen, and anti-HCV) and signs of infection (fever and chills) in patients with an established history of drug toxicity. The following blood tests were performed at the time of admission: hemoglobin, platelet count, prothrombin time (PT), serum aspartate aminotransferase (AST), serum alanine aminotransferase (ALT), and serum total bilirubin. The clinical characteristics of the AHA and ATH patients at the peak of hepatitis are shown in Table 1.

\section{Isolation of peripheral blood mononuclear cells}

Peripheral blood mononuclear cells (PBMCs) were isolated by Ficoll-Histopaque (Sigma Chemical, St. Louis, MO, USA) density centrifugation and cryopreserved as described previously. ${ }^{9}$

\section{Immunophenotyping by flow cytometry}

All fluorescent monoclonal antibodies were purchased from BD Bioscience (San Jose, CA, USA). The cells were stained with fluorescent antibodies according to the manufacturer's instructions. Briefly, PBMCs $\left(1 \times 10^{6}\right)$ from the patients were incubated with the fluorescent antibodies, washed three times, and then fixed with 2\% paraformaldehyde (Sigma-Aldrich, St. Louis, MO, USA). The cells were then subjected to flow cytometry on a FACSCanto (Becton Dickinson, San Jose, CA, USA) and analyzed using FlowJo (Tree Star, Ashland, OR, USA), gating on live lymphoid cells based on forward and side scatter profiles, and 7-amino-actinomycin D staining. Compensations were established using single-color controls. Staining for the intracellular expression of CTLA-4 was done on permeabilized cells. PD-1 and CTLA-4 positivities were determined by comparison with the defined cutoff values obtained with unstained control cells (PD-1: 99.5\%, CTLA-4: 99.9\%) as previously described. ${ }^{10,11}$

\section{Statistical analysis}

Clinical and immunological parameters in the different patient groups were compared using the nonparametric MannWhitney U test and Kruskal-Wallis test. Correlations were tested for statistical significance using the Spearman rank correlation. A p-value $<0.05$ was considered to indicate significance.

\section{RESULTS}

\section{Symptomatic AHA is associated with increased PD-1 and CTLA-4 expression}

PD-1 and/or CTLA-4 are overexpressed on the T-cells of patients with chronic HCV and HBV infections. ${ }^{7}$ We therefore examined whether the expression of these inhibitory receptors is similarly increased in T-cells from patients with acute hepatitis. As shown in Fig. 1, the expression of PD- 1 by CD8 and CD4 $\mathrm{T}$-cells was significantly higher $(\mathrm{p}=0.003$ and $\mathrm{p}=0.018$, respectively; Kruskal-Wallis test) in patients with symptomatic AHA than in either patients with nonviral ATH or healthy controls (Fig. 1A). CTLA-4 expression was also significantly upregulated in the CD8 cells of AHA patients, whereas in their CD4 cells there was a similar, albeit nonsignificant trend $(\mathrm{p}=0.0007$ and $\mathrm{p}=0.144$, respectively; Kruskal-Wallis tests) (Fig. 1B). These results showed that HAV infection is associated with the upregulation of the inhibitory T-cell costimulatory receptors PD-1 and CTLA-4, especially in CD8 T-cells.

\section{PD-1 expression correlates directly with that of CTLA-4 in symptomatic AHA}

In the group of AHA patients, PD-1 expression correlated strongly with the expression of CTLA- $4\left(R^{2}=0.859, p=0.001\right)$ (Fig. 2A, right). Figure $2 \mathrm{~B}$ shows the representative expression of PD- 1 and CTLA- 4 by CD4 T and CD8 T-cells. The preferential expression of CTLA-4 by PD-1-positive T-cells was consistent with the strong association between these two inhibitory T-cell

Table 1. Clinical Characteristics of Patients with Acute Hepatitis

\begin{tabular}{lccc} 
& $\begin{array}{c}\text { Nonviral acute toxic hepatitis } \\
(\mathrm{n}=6)\end{array}$ & $\begin{array}{c}\text { Symptomatic acute hepatitis A } \\
(\mathrm{n}=7)\end{array}$ & $\begin{array}{c}\text { Healthy control } \\
(\mathrm{n}=5)\end{array}$ \\
\hline Sex, male:female & $5: 1$ & $4: 3$ & $3: 2$ \\
Age, yr* & 36 & 34 & 32 \\
Peak AST, U/L* & 898 & 1,714 & 20 \\
Peak ALT, U/L* & 1,733 & 2,263 & 24 \\
Peak total bilirubin, $\mathrm{mg} / \mathrm{dL}^{*}$ & 2.85 & 4.08 & 0.8 \\
IgM anti-HAV & Negative & Positive & ND \\
\hline
\end{tabular}

AST, serum aspartate aminotransferase; ALT, serum alanine aminotransferase; HAV, hepatitis A virus; ND, not determined. *Median values. 

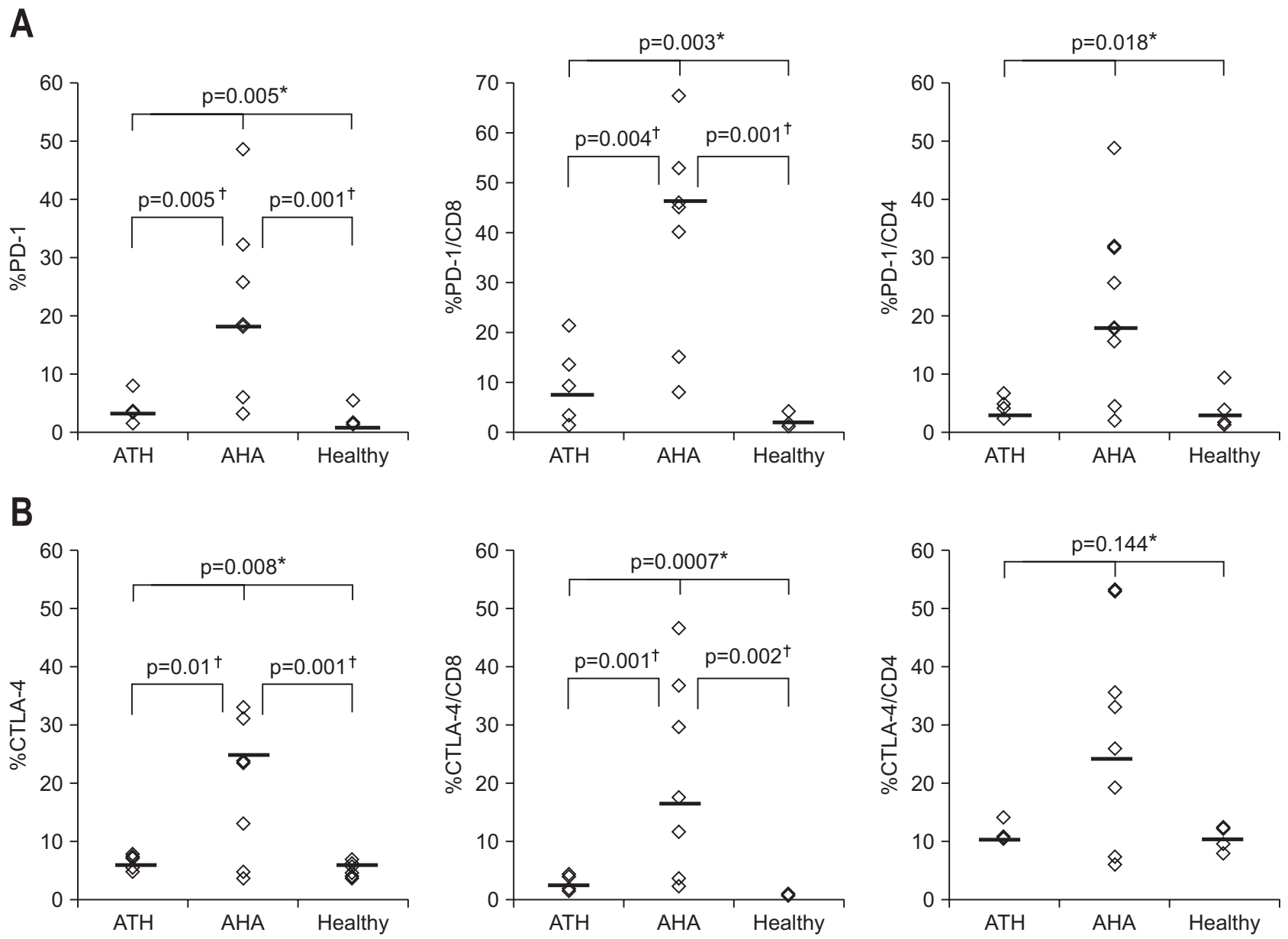

Fig. 1. Increased frequencies of programmed death 1 (PD-1)+ or cytotoxic $\mathrm{T}$ lymphocyte antigen 4 (CTLA-4)+ T-cells from patients with symptomatic acute hepatitis A (AHA) compared to nonviral acute toxic hepatitis (ATH). (A) \%PD-1+ (median: ATH, 3.6; AHA, 18.3; healthy, 1.6), \%PD1+/CD8, and \%PD-1+/CD4 T-cells from six patients with nonviral ATH, seven patients with AHA, and five healthy controls. (B) \%CTLA-4+ (median: ATH, 6.8; AHA, 23.5; healthy, 5.9), \%CTLA-4+/CD8, and \%CTLA-4+/CD4 T-cells from patients with nonviral ATH, AHA, and in healthy controls. *p-values by Kruskal-Wallis; ${ }^{\dagger}$ p-values by Mann-Whitney U test.

receptors.

\section{PD-1 and CTLA-4 expression decreases dramatically during the convalescent phase of AHA}

We monitored the expression of PD-1 and CTLA-4 from the symptomatic phase until the end of the convalescent phase in patients with acute hepatitis. PD-1 and CTLA-4 were highly upregulated during the symptomatic phase in patients with AHA but both decreased rapidly as these patients entered the convalescent phase (Fig. 3A, right and B, right), suggesting a strong relationship between the clinical manifestations of AHA and the expression of immune inhibitory receptors of antiviral effector T-cells, such as PD-1 and CTLA-4.

\section{CTLA-4 expression correlates with clinical parameters of liver injury in patients with acute hepatitis}

PD-1 expression on T-cells was not associated significantly with clinical parameters of liver injury, such as AST and ALT, in either AHA or ATH patients. Conversely, in both groups the expression of CTLA-4 correlated significantly with the levels of AST $\left(R^{2}=0.295, p=0.0337\right)$ (Fig. $4 A$, far left) and ALT $\left(R^{2}=0.246\right.$, $\mathrm{p}=0.0419$ ) (Fig. $4 \mathrm{~B}$, far left). According to subgroups such as AHA and ATH, these correlations between CTLA- 4 and AST or ALT were not significant, because of small sample size.

\section{DISCUSSION}

The overexpression of PD- 1 and CTLA- 4 by antigen-specific T-cells from patients with chronic viral infections, including $\mathrm{HIV}, \mathrm{HBV}$, and HCV, has been demonstrated in several studies. The expression of these immune inhibitory receptors was shown to be associated with the functional impairment of T-cells, ${ }^{5,12,13}$ suggesting that the prolonged expression of PD-1 and CTLA4 is related to the chronicity of viral forms of hepatitis. In this study, we asked whether PD-1 and CTLA- 4 were expressed by the T-cells of patients with AHA, which is a self-limited, acute, 

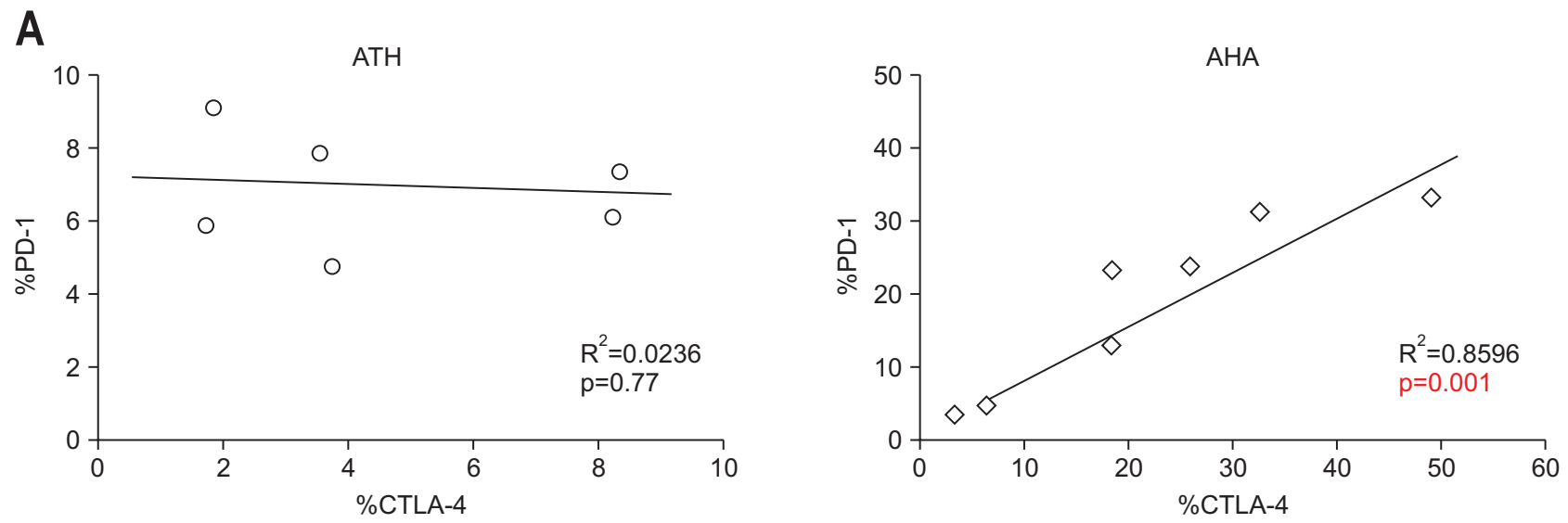

B

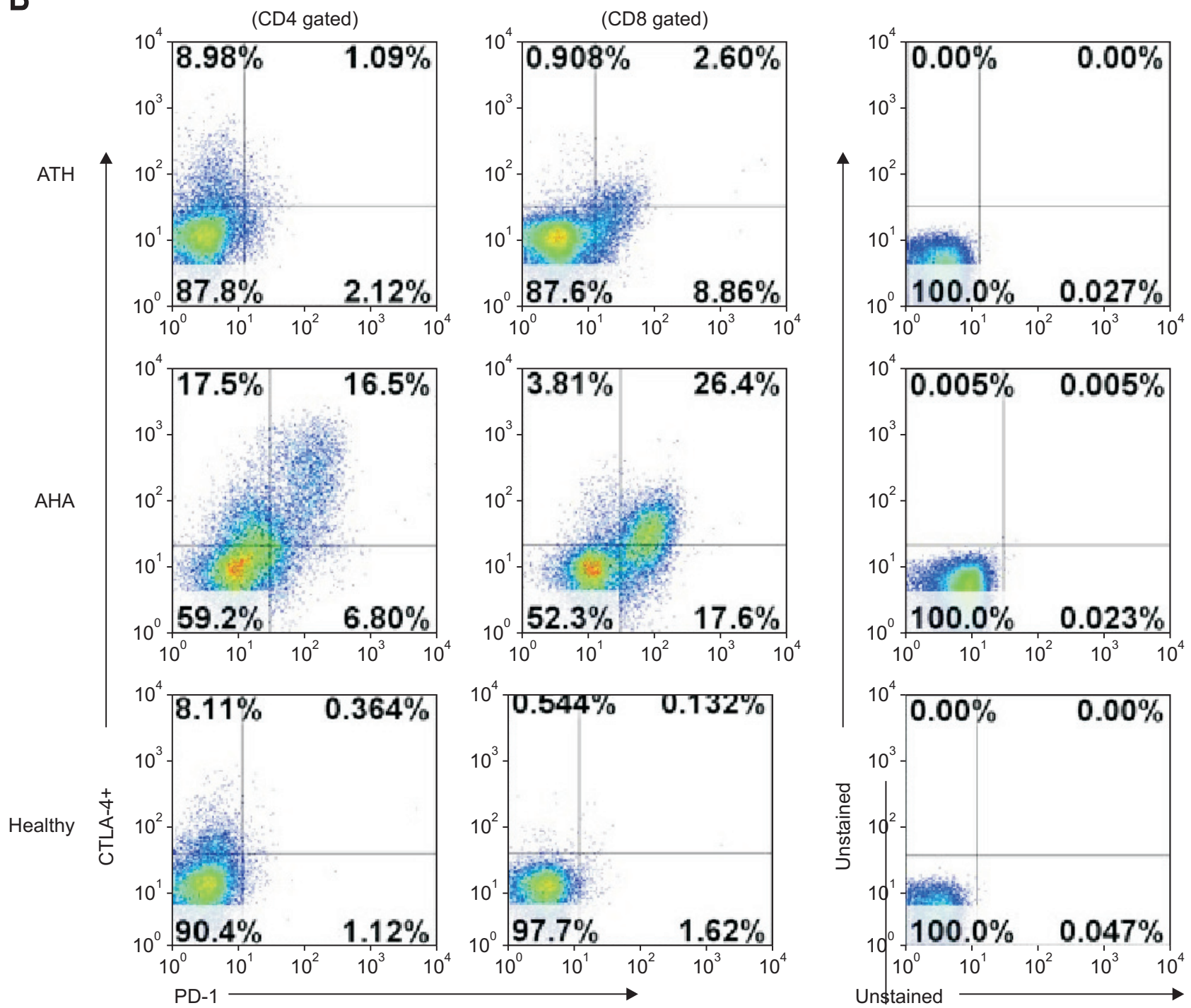

Fig. 2. Direct correlation between programmed death 1 (PD-1) and cytotoxic T lymphocyte antigen 4 (CTLA-4) expression in symptomatic acute hepatitis A (AHA). (A) Correlation between PD-1 and CTLA-4 expression in nonviral acute toxic hepatitis (ATH) and in AHA. (B) Representative PD-1 and CTLA-4 expression on CD4+ and CD8+ T-cells. 


\section{A}

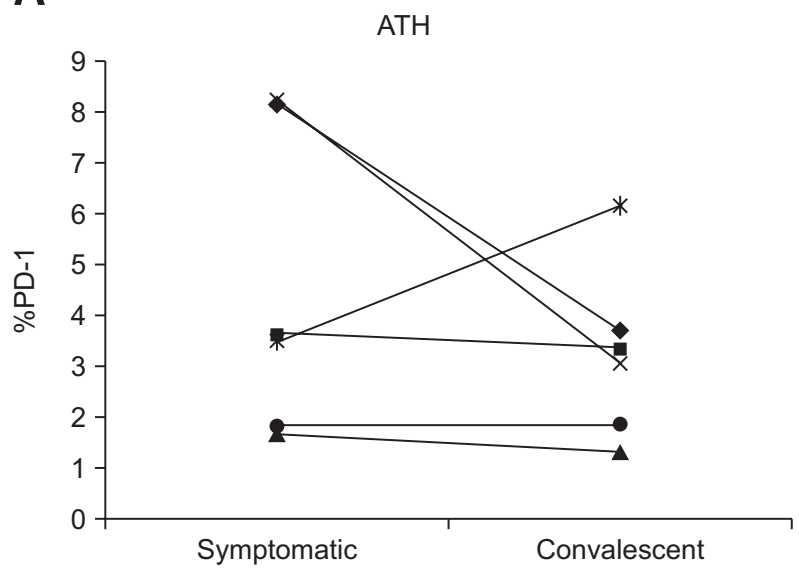

B

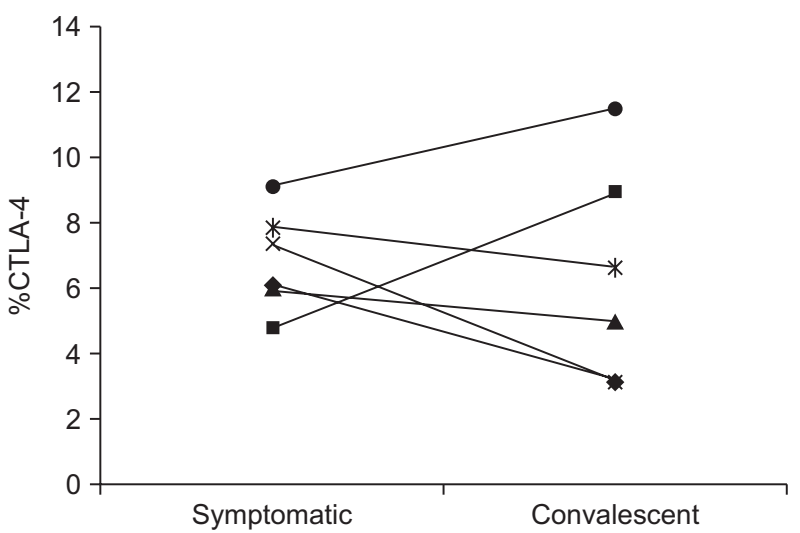

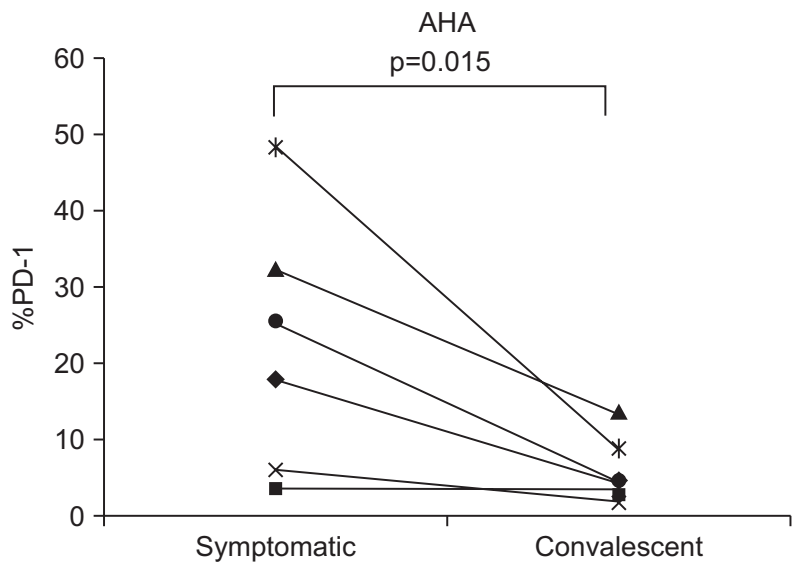

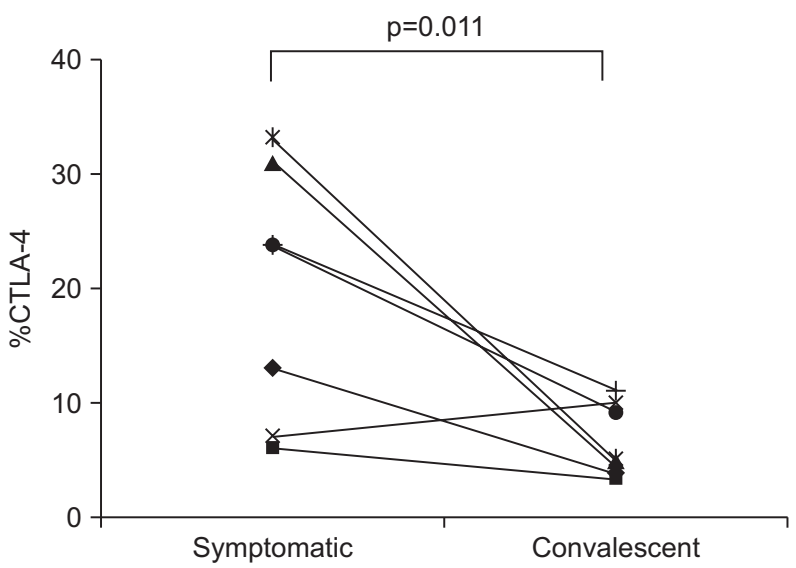

Fig. 3. Decreased programmed death 1 (PD-1) and cytotoxic T lymphocyte antigen 4 (CTLA-4) in the convalescent phase of acute hepatitis A (AHA). (A) \%PD-1+ during the symptomatic and convalescent phases of nonviral acute toxic hepatitis (ATH) and AHA. (B) \%CTLA-4+ during the symptomatic and convalescent phases of nonviral ATH and AHA.

viral hepatitis. Indeed, T-cells from patients with AHA expressed significantly higher levels of PD-1 and CTLA-4 than cells from healthy controls. Interestingly, neither PD-1 nor CTLA-4 expression was increased in T-cells from patients with ATH; in fact, expression was similar to that in healthy controls. Our study is the first to compare PD-1 and CTLA-4 expression in AHA and ATH. The results suggest that the expression of these immune inhibitory receptors in hepatitis is related either to the etiology of the disease or to their induction by viral factors. The strong correlation between PD-1 and CTLA-4 expression in AHA (Fig $2 \mathrm{~A}$, right) indicated that they are simultaneously expressed on the surface of T-cells from these patients. Similar results were obtained by Nakamoto et al. ${ }^{4}$ regarding the expression of PD-1 and CTLA-4 on T-cells from patients with chronic hepatitis C. In addition, while both PD-1 and CTLA-4 were highly upregulated during the symptomatic phase of AHA, their expressions were dramatically reduced to the control level during the recovery phase. The Change of PD-1 or CTLA- 4 could be associated with the changes of antiviral immune response in other viral diseases caused by HBV and HCV. ${ }^{11,14}$ Therefore, we hypothesized that the expression of PD-1 and CTLA-4 may correlate with clinical liver injury parameters such as AST and ALT. However, only the expression of CTLA-4, and not that of PD-1, was associated with the clinical parameters of liver injury, specifically, AST and ALT. In a recent report by Wang et al., ${ }^{15}$ the level of PD-1 correlated significantly with serum ALT in patients with chronic HBV infection. Thus, PD-1 and CTLA-4 could serve as useful biomarkers of the severity of liver injury, although we were not able to correlate the level of PD-1 with either AST or ALT, possibly because of the small sample size. Taken together our findings of the changing expression of PD-1 and CTLA-4 during the symptomatic and recovery phases of AHA point to the protective effects of these inhibitory molecules, perhaps by suppressing the activity of cytotoxic T-cells, thereby preventing the induced fulminant destruction of HAV-infected hepatocytes.

\section{CONFLICTS OF INTEREST}

No potential conflict of interest relevant to this article was reported. 

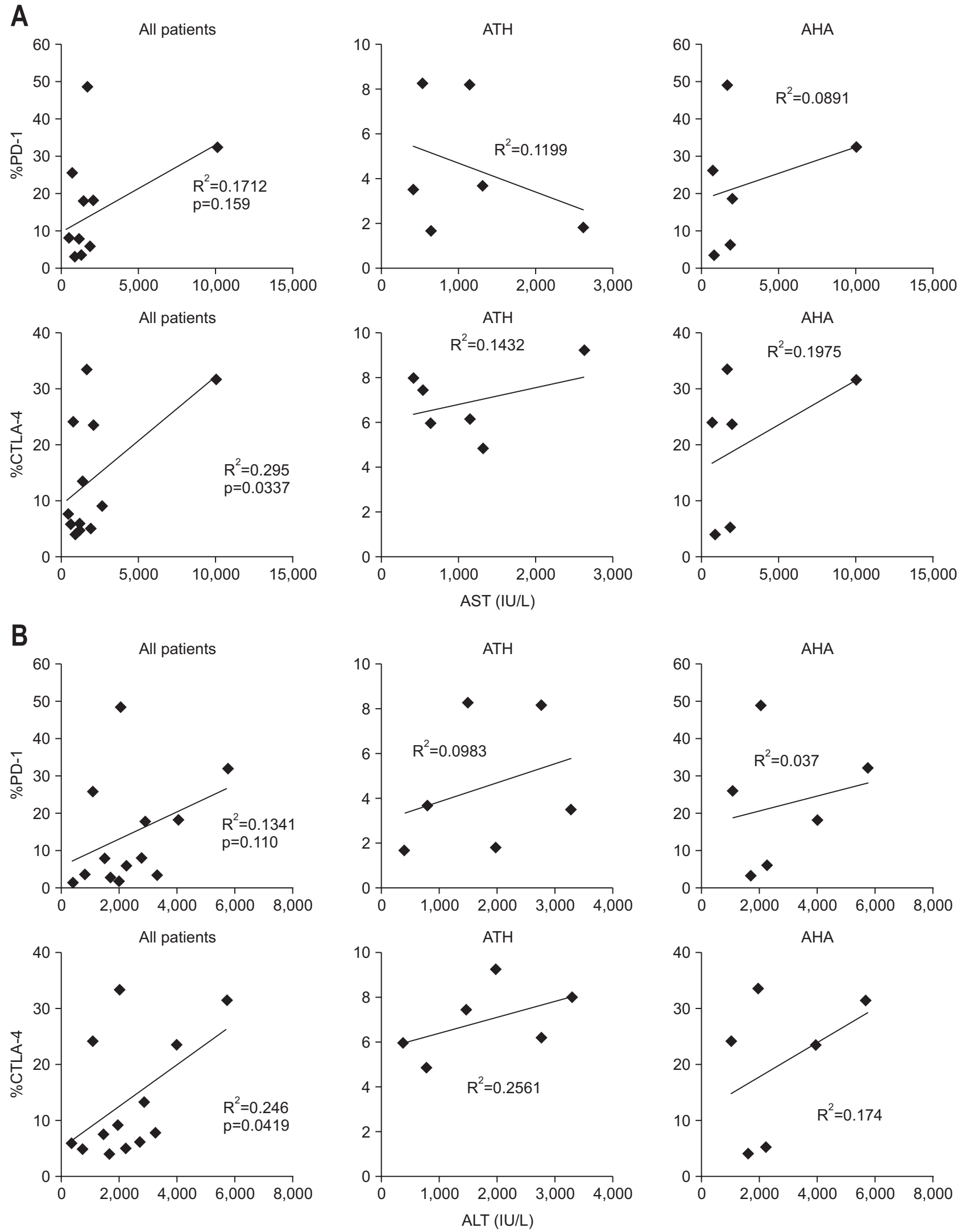

Fig. 4. Correlation between programmed death 1 (PD-1) expression, cytotoxic T lymphocyte antigen 4 (CTLA-4) expression and clinical liver injury parameters such as aspartate aminotransferase (AST) and alanine aminotransferase (ALT) in all patients and in patients with nonviral acute toxic hepatitis (ATH) or acute hepatitis A (AHA). (A) Correlation between \%PD-1 and \%CTLA-4 T-cells and AST among all patients, patients with nonviral ATH and patients with AHA. (B) Correlation between \%PD-1 and \%CTLA-4 T-cells and ALT among all patients, patients with nonviral ATH and patients with AHA. The healthy controls were excluded. 


\section{ACKNOWLEDGEMENTS}

The authors wish to acknowledge the financial support of the Catholic Medical Center Research Foundation made in the program year of 2012 .

\section{REFERENCES}

1. De Filippis P, Divizia M, Mele A, Adamo B, Panà A. Detection of hepatitis A virus in the stools of healthy people from endemic areas. Eur J Epidemiol 1987;3:172-175.

2. Kwon SY. Current status of liver diseases in Korea: hepatitis A. Korean J Hepatol 2009;15 Suppl 6:S7-S12.

3. Fife BT, Bluestone JA. Control of peripheral T-cell tolerance and autoimmunity via the CTLA-4 and PD-1 pathways. Immunol Rev 2008;224:166-182.

4. Nakamoto N, Cho H, Shaked A, et al. Synergistic reversal of intrahepatic HCV-specific CD8 T cell exhaustion by combined PD-1/ CTLA-4 blockade. PLoS Pathog 2009;5:e1000313.

5. Evans A, Riva A, Cooksley H, et al. Programmed death 1 expression during antiviral treatment of chronic hepatitis B: impact of hepatitis B e-antigen seroconversion. Hepatology 2008;48:759769.

6. Day CL, Kaufmann DE, Kiepiela P, et al. PD-1 expression on HIVspecific T cells is associated with T-cell exhaustion and disease progression. Nature 2006;443:350-354.

7. Kaufmann DE, Walker BD. PD-1 and CTLA-4 inhibitory cosignaling pathways in HIV infection and the potential for therapeutic intervention. J Immunol 2009;182:5891-5897.

8. Elrefaei M, Baker CA, Jones NG, Bangsberg DR, Cao H. Pres- ence of suppressor HIV-specific CD8+ T cells is associated with increased PD-1 expression on effector CD8+ T cells. J Immunol 2008;180:7757-7763.

9. Sugimoto K, Ikeda F, Stadanlick J, Nunes FA, Alter HJ, Chang KM. Suppression of HCV-specific T cells without differential hierarchy demonstrated ex vivo in persistent HCV infection. Hepatology 2003;38:1437-1448.

10. Ebinuma H, Nakamoto N, Li Y, et al. Identification and in vitro expansion of functional antigen-specific CD25+ FoxP3+ regulatory T cells in hepatitis C virus infection. J Virol 2008;82:50435053.

11. Nakamoto N, Kaplan DE, Coleclough J, et al. Functional restoration of HCV-specific CD8 T cells by PD-1 blockade is defined by PD-1 expression and compartmentalization. Gastroenterology 2008;134:1927-1937.e2.

12. Freeman GJ, Wherry EJ, Ahmed R, Sharpe AH. Reinvigorating exhausted HIV-specific T cells via PD-1-PD-1 ligand blockade. J Exp Med 2006;203:2223-2227.

13. Golden-Mason L, Palmer B, Klarquist J, Mengshol JA, Castelblanco N, Rosen HR. Upregulation of PD-1 expression on circulating and intrahepatic hepatitis C virus-specific CD8+ T cells associated with reversible immune dysfunction. J Virol 2007;81:9249-9258.

14. Wenjin Z, Chuanhui P, Yunle W, Lateef SA, Shusen Z. Longitudinal fluctuations in PD1 and PD-L1 expression in association with changes in anti-viral immune response in chronic hepatitis B. BMC Gastroenterol 2012;12:109.

15. Wang L, Zhao C, Peng Q, Shi J, Gu G. Expression levels of CD28, CTLA-4, PD-1 and Tim-3 as novel indicators of T-cell immune function in patients with chronic hepatitis B virus infection. Biomed Rep 2014;2:270-274. 